\title{
Usability Issues in City Tourism Website Design: A Content Analysis
}

\author{
Quan Zhou \\ University of Washington \\ qzhou@u.washington.edu
}

\author{
Rich DeSantis \\ University of Washington \\ hci@u.washington.edu
}

\begin{abstract}
This paper addresses usability issues in city tourism website design. Through content analysis, the paper examines the content and functions of fifty-five city tourism websites covering North America, Europe, Asia, Australia and Africa. The paper analyzes the characteristics of city tourism websites and target users. It generalizes the existing literature and identifies key factors influencing city tourism website usability. The paper focuses on usability challenges and analyzes cross-cultural differences on city tourism websites. It reports the content analysis data and provides suggestions for making tourism websites more effective, efficient and easy-to-use.
\end{abstract}

Keywords: usability, city tourism website, content analysis, cross-cultural

\section{Introduction}

The dramatic development of Information and Communication Technology (ICT) has changed the way people search for and digest information. As the World Wide Web became prevalent around the globe, more travelers used the Internet as a medium for searching tourism information and planning their trips.

Today, tourism information is among the most popular and frequently visited information on the Internet. It is information requested by people from different countries and cultures; and it is information nearly everyone needs from time to time. "Information is a basic aspect of the product of the tourist industry, and the use of ICT has been at the core of the way it has been structured in the last few years." [1] The domain of traveling plays "a major role in each culture" and is "deeply anchored in the local culture." [2]

Tourism websites play an indispensable role in people's traveling decision-making before their departure. Tourism research has shown that although travelers make many different kinds of decisions, "central decisions are made at the beginning of travel planning and usually hard to change." [3] [4] [5] In this sense, the attractiveness of tourism websites has important impact on travelers' choices.

Buhalis [6] advocated: "information technology will be instrumental in the industries' ability to enhance their future efficiency and strategic competitiveness." [7] Internet technology not only offers dynamic options for travelers, but also leads the tourism industry to build "homes" on the Internet in order to market their services. The multimedia and interactivity of the web has tremendous influence on tourism. Research [7] argued that because the large volume of information that multimedia carries are disseminated over long distances immediately, the multimedia has "substantially improved the effectiveness and efficiency of information presentation and dissemination through electronic channels."

While the depth and scope of tourism website services have expanded, the usability of the tourism websites remains a problem. Many of them have poor usability, weak functions, inaccessibility, hard-to-use content and few crosscultural considerations. As Pan [5] mentioned, research projects conducted by Radosevich [8] and Stoltz (1999) have confirmed: "trip planning on the Web is a frustrating experience." Studies also found that the usability problems of Internet tourism information are closely connected to 
travelers' background knowledge and individual demand. [4] [5]

Unlike other research that focuses on the travel booking system or tourism information architecture, this paper takes a unique look at city tourism websites and the usability of their content and functions. The study examines 55 city tourism websites in North America, Europe, Asia, Australia/New Zealand and Africa. Through content analysis, the paper identifies commonalities, discrepancies and patterns of the content and functions and provides suggestions to improve city tourism websites' usability.

\section{Literature Review}

A literature search shows that research done in this field is generally concerned with the following aspects: tourism information systems \& architecture [10], data warehousing [11], electronic tourism commerce [12], information search [5], and interface design [13], etc. Significant efforts have been focused on the tourism information system, electronic commerce and value chain. [14]

There are case studies in terms of regional tourism. For instance, Friel and colleagues [15] examined service dimensions of travel distribution in India. Daniele and colleagues [14] explored a destination marketing system for Australia's national tourism data warehouse. Some usability issues are also covered in the existing studies. Crichton and Frew [13] focused on the usability of reservations systems on travel websites. Research [16] [17] [18] has also extensively examined typology of tourism websites and travel information search.

Due to internationalization and globalization attributes in the tourism industry, tourism website usability is closely connected with cross-cultural context. Recent research showed that people's demands regarding the design of user interfaces are influenced by the different cultures they come from. [19] [2] For instance, studies have shown that a hotel's date entry form design needs to accommodate the different data formats (e.g. in the U.S. it's MM-DD-YYYY; in Japan it's YYYYMM-DD) [20]. "Cross-cultural design should be considered at the beginning of the design process and not at the end, such as simply translating the content into another language." [2] Degen and colleagues' [2] cross-cultural study revealed that including user input of different cultures from the initial phase of the design is very necessary. "Cosmetic changes such as translating the language or changing the colors, instituted late in the process of design or even after the fact, are insufficient.” [2]

The multimedia function of the Internet is also a focus of the literature. Research [21] indicated that one important part of tourism marketing is "experience-oriented tourism" which "emphasizes activity, events, and fantastic or exotic experiences." Functions such as virtual tour, gallery, or interactive map can help potential tourists to develop expectations about what they can experience at the destination. "Interactivity and multimedia are key factors to provide virtual experiences." [21] Cho and Fesenmaier's [21] research advocated that "the richness of sensory information" and "the interactivity between human and virtual environment" are two factors crucial to travel planning and they concluded that a virtual tour can "enhance the memory of destination" and can help users "form more vivid and clear destination image." It puts tourists "in a better position to choose a destination" because the virtual experience simulates experiential information. [21]

Studies have also explored the factors that correlate with the success of tourism website design. Degen and Pedell's [22] research investigated several design factors that could be used to assess specific elements of interface design. These factors include content, function, layout, linkage, wording and media. [22] [2] In addition, Degen et al.'s (2005) study focused specifically on examining these factors with crosscultural differences. In our content analysis study, we primarily focus on the usability of content and functions with some analysis on linkage, wording and media.

Studies have found that many travelers look for an "information portal" as an "authoritative" web page such as a city's Convention and Visitor Bureau website. [18] Hence, most of the city tourism websites we examined were the ones usually visited by the travelers. This prompts a thorough examination of the city tourism website.

Despite the considerable amount of research in ICT in tourism and hospitality, little research has been reported on the usability, functionality and accessibility of the tourism website design. In 
addition, few studies have examined the crosscultural context. City tourism websites, an important part of online travel resources, are a relatively "empty" field in need of exploration.

\section{City Tourism Website}

Because they are different from other types of websites, tourism websites have their own unique characteristics.

\section{Features of Tourism Websites}

The purpose of tourism websites is usually to engage the target users and to provide convenient traveler-centered information.

Since a lot of travelers come from foreign countries and regions, tourism websites should accommodate cultural differences and have sufficient understanding to provide services for users in different time zones with different languages, cultures, currencies, and customs.

Given this situation, the following factors are highly influential to the success of a tourism website: direction information, accessibility to comprehensive coverage of local information, interactivity and vivid description and accommodation of multilingual and multicultural user population.

\section{Target Users}

Travelers come to a tourism website to learn from traveling resources that help them make travel decisions and plans. They could come from the other side of the world, knowing nothing about their destinations. As a consequence, they need appropriately presented information to orient them.

Travelers usually need vivid and comprehensive information (e.g. yellow pages), which will satisfy their information need, budget estimate, itinerary planning, etc. They have a great need for directions, hence, an interactive and easy-to-use map or similar tool is crucial for them to learn indepth and to better anticipate how they will navigate their traveling routes. The quality of images (e.g. pictures of destinations) or virtual tours is also very helpful. In addition, travelers also expect wording and terminology that is easy to understand. Since not all travelers understand the foreign language, a multilingual interface is a big plus.

\section{Methodology}

In order to have a thorough view of the usability of current city tourism websites, this study examines 55 sample city tourism websites. These 55 city tourism websites were selected from Yahoo's "travel" directory. (http://dir.yahoo.com/Recreation/Travel/)

The study tries to cover a diversity of tourism websites and thus to have a more persuasive and logical interpretation of the results. The sample websites come from North America, Europe, Asia, Australia and Africa. We included capital cities, big cities and smaller cities' websites, many with distinct functions and services.

In order to examine the content and function of these websites, we used content analysis methodology. "Content analysis is a research tool focusing on the actual content and internal features of media" (http://www.gslis.utexas.edu/ palmquis/cour ses/content.html). "It is used to determine the presence of certain words, concepts, themes, phrases, characters, or sentences within texts or sets of texts and to quantify this presence in an objective manner" (http://www.gslis.utexas.edu/ palmquis/courses/cont ent.html ). In Bernard Berelson's definition [23], content analysis is "a research technique for the objective, systematic, and quantitative description of manifest content of communications." Content analysis has also been expanded to examine webbased information and cultural values. [24] Hence, content analysis can be an effective tool to examine the content and functions of city tourism websites.

The 55 websites were grouped into five categories by regions: United States (US), Canada (CA), Europe (EU), Australian/New Zealand (AZ), Asia, and Africa (AF).

In order to keep a consistency and compatibility among these websites, an equal number of 10 websites were chosen for each category except Africa. The researchers were not able to find 10 African tourism websites that were the city's official sites. Instead, we found five African "national" tourism websites and used those in the study.

The homepage of each of the 55 websites was thoroughly examined and the data were recorded in terms of functions provided, services offered and content presented. The results were grouped in several ways: by nations, by attributes, etc. These data were analyzed using Microsoft Excel and 
SPSS (a statistical analysis software). The two coders coded the websites together in order to maintain rigor in the inter-coder reliability.

\section{Case Study of City Tourism Websites}

Fifty-five city tourism websites were analyzed considering the following aspects. They suggest the existing challenges on website usability and reveal the distinctions among different countries.

\section{Direction Information}

Mapping and direction are crucial information for city tourism websites. Maps give the visitors a useful overview for their travel planning, often vital for budget and time estimation. The study found that $80 \%$ of the sampled websites offered some type of map.

Travelers prefer to navigate the map in a way that helps them arrange their itinerary. One important feature of an efficient and functional map is interactivity. For instance, they want to be able to zoom, move, and search for elements on the map. It could be a street name, a building's picture, a driving guide, or a time estimate for a distance. For instance, San Francisco's website (http://www.sfvisitor.org) has many interactive maps concerning different districts. On Hong Kong's website (http://www.discoverhongkong.com), users could choose "Day" and "Night" on the map in order to see corresponding pictures. We found that out of the 55 websites, $45 \%$ offered a highly functional, interactive map allowing the user to re-center and zoom by clicking directly on the map graphic.

Many websites did not offer an interactive map (see Figure 1). Some of the offered maps are even too vague or small to read/print. One phenomenon we uncovered is that, none of the Australian, New Zealand or African websites examined offer interactive maps.

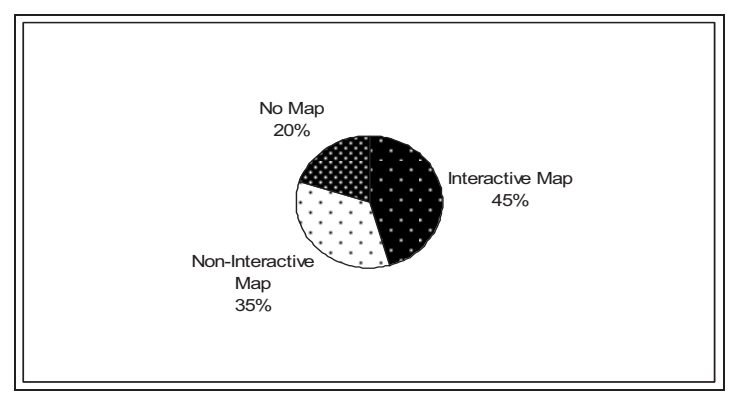

Figure 1. The distribution of sampled websites with interactive maps, non-interactive maps or no maps.

\section{Visa and Policy Information}

In a time when worldwide travel is common, visa and foreign policy information is a planning prerequisite. Hence, visa and policy information is frequently requested by visitors and access to this information becomes a criterion for the usability of tourism websites. This study found many city tourism websites ignoring visa and policy information. Asian and European city websites tend to provide visa information more than North American and Australian websites (see Table 1). Among the U.S. websites we examined, only 1 of them provides visa information even though the U.S. has one of the most complicated visa requirements. The same $10 \%$ frequency occurs on Canadian websites.

Table 1. Percentage of City Websites Providing Visa Information

\begin{tabular}{|c|c|c|c|c|c|}
\hline EU & Asia & US & CA & AZ & AF \\
\hline $\mathbf{4 0} \%$ & $\mathbf{4 0} \%$ & $\mathbf{1 0} \%$ & $\mathbf{1 0} \%$ & $\mathbf{1 0} \%$ & $\mathbf{2 0} \%$ \\
\hline
\end{tabular}

EU-Europe, CA-Canada, AZ-Australia/New Zealand, AF-Africa

\section{Multilingual Functions}

The international attribute of tourism indicates that multilingual services can improve a tourism website's usability and thus help people with language barriers.

Our observation revealed a significantly higher occurrence of language translations on Asian sites when compared with all other sampled regions (see Table 2). This is consistent with the region's rising economy and increasing interest in the tourism industry.

It is valuable to report that some websites are deeply internationalized and localized. For instance, Hong Kong's official tourism website (http://www.discoverhongkong.com) offers dynamic localization choices for travelers from another country. At least 12 different language interfaces are offered. Surprisingly, it offers six different types of English versions, such as American English, Australian English, etc. We also found that although many other websites only offer Simplified Chinese language (e.g. Berlin's city tourism website: http://www.berlin-touristinformation.de/index.en.html), Hong Kong's website provide both Simplified and Traditional Chinese. In addition, Hong Kong's website provides customizable visa and tour information for people from different countries. In fact, the interface and content of different languages are 
also different. For instance, the American English version has resources specifically for U.S. citizens.

Table 2. Percentage of Websites with Multilingual Support

\begin{tabular}{|c|c|l|l|l|l|}
\hline EU & Asia & US & CA & AZ & AF \\
\hline $\mathbf{3 0 \%}$ & $\mathbf{7 0 \%}$ & $\mathbf{4 0 \%}$ & $\mathbf{4 0 \%}$ & $\mathbf{4 0 \%}$ & $\mathbf{0 \%}$ \\
\hline
\end{tabular}

EU-Europe, CA-Canada, AZ-Australia/New Zealand, AF-Africa

\section{Gallery and Image Presentation}

The tourism information design literature indicates that attractive photo galleries can stimulate travel planners' interests. Beautiful photos seem to increase the satisfaction level of the user's experience. Of the 55 sites reviewed for this study, 33 of them, or $60 \%$, included a photo gallery. With the exception of Europe at $40 \%$, and Australia at 50\%, the majority of the sampled region's sites included a gallery. Asian site had the largest percentage of sites with photo galleries at $70 \%$ (see Table 3 ).

Table 3. Percentage of Websites with a Photo Gallery

\begin{tabular}{|c|c|c|c|c|c|}
\hline EU & Asia & US & CA & AZ & AF \\
\hline $\mathbf{4 0} \%$ & $\mathbf{7 0} \%$ & $\mathbf{6 0} \%$ & $\mathbf{6 0} \%$ & $\mathbf{5 0} \%$ & $\mathbf{6 0 \%}$ \\
\hline
\end{tabular}

\section{Currency Considerations}

Currency exchange rates can affect a traveler's plans and lead them to vacation in locations where their currency is strong. In addition travelers like to be able to calculate the relative costs of their travel essentials like hotels and rental cars.

Some of the reviewed sites included their own currency exchange calculator while others provided a link to the "The Universal Currency Converter" at http://www.xe.com/ucc. It is interesting to learn that none of the sampled U.S. websites offers currency converter. Within the North American region, Canada shows a significant difference from the United States in this aspect (see Table 4).

Table 4. Percentage of Websites with Currency Support

\begin{tabular}{|c|c|c|c|c|c|}
\hline EU & Asia & US & CA & AZ & AF \\
\hline $\mathbf{4 0 \%}$ & $\mathbf{5 0} \%$ & $\mathbf{0 \%}$ & $\mathbf{5 0} \%$ & $\mathbf{2 0} \%$ & $\mathbf{4 0} \%$ \\
\hline
\end{tabular}

EU-Europe, CA-Canada, AZ-Australia/New Zealand, AF-Africa

\section{Local Information (Weather/Time)}

To insure the best possible experience in a new city, travelers must plan ahead by researching the climate and weather conditions of their destination.
Of the sampled sites, many sites included both typical climate and weather by month and the current climate and weather.

Time is another important thing that travelers need to know in order to plan their detail schedule. None of the six categories we examined have more than $50 \%$ of the websites showing local time (see Figure 2). Particularly, no sampled Canadian websites provide local time or link to time converters.

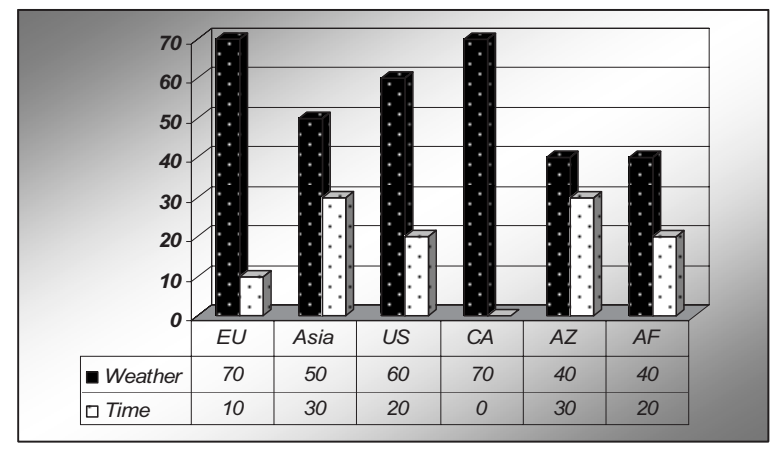

EU-Europe, CA-Canada, AZ-Australia/New Zealand, AF-Africa Figure 2. Percentages of websites showing local time/weather.

\section{Utility Functions}

Information Design research shows that utility functions are critical for the ease of website accessibility. [25] Many types of utility links: sitemap/index, and search engines are commonly seen on websites.

Except for African websites, all other countries have a certain number of websites that include sitemap and search. U.S. websites show a more balanced consideration between sitemap and search than Australian and New Zealand websites (see Figure 3).

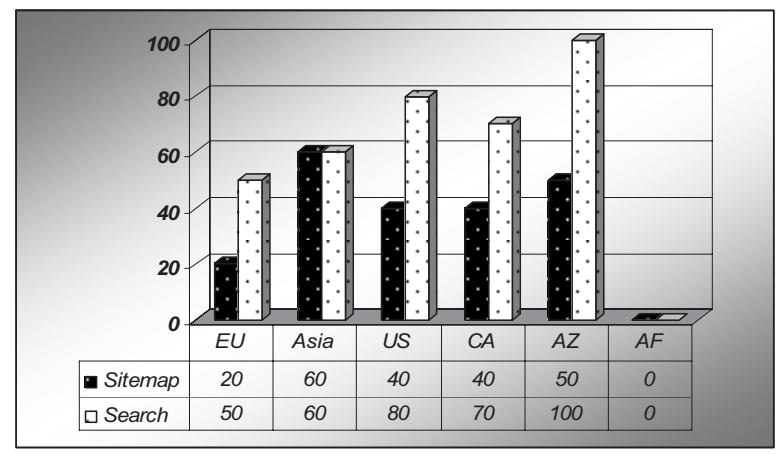

EU-Europe, CA-Canada, AZ-Australia/New Zealand, AF-Africa

Figure 3. Percentages of websites offering sitemap and search. 
Although some websites have search functions, some of them are not as functional as they are supposed to be. For instance, Winnipeg city's tourism website (http://www.tourism.winnipeg.mb.ca) asks the user to search for more than three characters. Yet, there are no results when we searched "map" or "maps".

\section{Yellow Pages Information}

When travelers want to find some information about the local city, the first resource that comes to their mind is probably yellow pages or directory. As shown in Table 5, we found that more U.S. and African websites have yellow page links than other regions. Although African websites are found weak in some other aspects, they do well in providing yellow page information.

Table 5. Percentage of Websites with Yellow Page Links

\begin{tabular}{|c|c|c|c|c|c|}
\hline EU & Asia & US & CA & AZ & AF \\
\hline $\mathbf{3 0} \%$ & $\mathbf{4 0} \%$ & $\mathbf{6 0} \%$ & $\mathbf{4 0} \%$ & $\mathbf{3 0} \%$ & $\mathbf{6 0} \%$ \\
\hline
\end{tabular}

EU-Europe, CA-Canada, AZ-Australia/New Zealand, AF-Africa

\section{Adoption of Multimedia Technologies} A lot of multimedia technologies are found on the examined websites. Specifically, we found that Asian websites (e.g., Korea's Busan city tourism website: http://www.visit.busan.kr/eng) tend to use more Flash animations than North American and European websites. African websites are generally lacking multimedia functions.

\section{Wording \& Terminology}

While most websites examined have basically similar wording and terminology, we found some exceptions which created confusions. For instance, Osaka's website (http://www.octb.jp/english) uses "Access" to represent "Transportation". Nonetheless, "access" could be interpreted as "accessibility" or information for disabled people. This is caused by the different habits of language.

\section{Content Categorization}

This study found that U.S. city tourism websites have strengths in tourism information categorization. For most U.S. websites examined, the designers used a two dimensional approach in classifying the content. One dimension is useroriented navigation. For instance, many websites (e.g. Chicago's website: http://www.chicago.il.org) have menus for "Visitors", "Meeting Professionals", "Media", each linking to different content and resources that target audiences need. The other dimension is according to the information need such as "Accommodation", "Sightseeing", "Transportation", etc. Through this two dimensional approach, users' needs are represented and thoroughly helped.

\section{Suggestions}

This study shows various facets of city tourism website usability. It reveals the apparent differences among countries of different economic level as well as culture. Based on the results, we suggest the following solutions to improve the usability of city tourism websites and to bridge the gap among countries.

- Implementing digital interactive mapping.

- Providing visa/policy and currency converting information.

- Presenting local information such as weather and time.

- Having sitemap or A-Z index as well as valid search.

- Facilitating website localization. Multilingual services should be provided to reduce language barriers on tourism. Presenting information for specific users would also help them easily find the information they need and build trust between the website and the users.

- Broadening the way to engage the users. Encouraging Image exhibition/gallery especially increasing interactivity and "fun-ability"

- Planning for cross-cultural design at the beginning of the city tourism website design and throughout the project.

- Using a persona in the design process to facilitate traveler-centered design.

- Surveying users from different target populations before tourism website design and doing iterative testing and evaluation during the design process.

\section{Conclusion}

This research takes a close look at representative tourism websites across North America, Europe, Asia, Australia/New Zealand, and Africa. It shows that usability challenges exist in various aspects in both content and functions. Many problems reflect a lack of user-centered design and interactivity in the city tourism website development. This research also found that usability is closely connected with cultural differences. A well- 
designed city tourism website should consider the distinct navigating and information seeking behaviors of different users which is especially vital in the international tourism industry. A digital divide was also found in city tourism websites among different countries.

\section{Limitations \& Future Research}

We wish we had more time in doing this research. One limitation lies in language barriers. We only included English language websites in this research. We also had a limited number of sample websites. Future research can be done in studying multi-lingual tourism website such as Chinese language websites, French tourism websites, etc. It is also timely and important to investigate how cultural differences influence the tourism information seeking patterns thus improving usability. Research on the principle of UserCentered Design is also needed in the tourism information design field.

\section{References}

[1] M. A. Rastrollo and P. Alarcon, " The Competitiveness of Traditional Tourist Destinations in the Information Economy," in Proc. of the International Conference on Information and Communication Technologies in Tourism 2000. New York: Springer-Verlag, pp. 209-217, 2000.

[2] H. Degen, K. L. Lubin, S. Pedell, and Z. Ji, "Travel Planning on the Web: A Cross-Cultural Case Study," in Usability and Internationalization of Information Technology, N. Aykin, Ed. Mahwah, NJ: Lawrence Erlbaum Associates, pp. 313-343, 2005.

[3] D. R. Fesenmaier and J. Jeng, "Assessing Structure in the Pleasure Trip Planning Process," Tourism Analysis, vol. 5, no. 1, pp. 13-29, 2000.

[4] J. Jeng, Exploring the Travel Planning Hierarchy: An Interactive Web Experiment. Dissertation. University of Illinois at UrbanaChampaign, 1999.

[5] Pan, B., Travel Information Search on the Internet: An Exploratory Study. Dissertation. University of Illinois at Urbana-Champaign, 2003.
[6] D. Buhalis, The Impact of Information Telecommunication Technologies on Tourism Channels: Implications for the Small and Medium Sized Tourism Enterprises. Dissertation. University of Surrey, Guildford, UK, 1995.

[7] M. Sigala, D. Airey, P. Jones, and A. Lockwood, "The Diffusion and Application of Multimedia Technologies in the Tourism and Hospitality Industries," in Proc. of the International Conference on Information and Communication Technologies in Tourism 2000. New York: Springer-Verlag, 2000, pp. 396-407.

[8] Radosevich, L., "Fixing Web-Site Usability," InforWorld, vol. 19, no. 50, pp. 81-82, 1997.

[9] Stoltz, C., "Each Year, a Bit Less," Washington Post, Nov. 11, 1999. Retrieved January 1, 2005 from http://www.hci.cornell.edu/bing/dissertation.pdf.

[10] J. Palkoska, A. Dunzendorfer, and J. Küng, "Vague Queries in Tourism Information Systems," in Proc. of the International Conference on Information and Communication Technologies in Tourism 2000, New York: Springer-Verlag, 2000, pp. 61-70.

[11] P. Sharma, D. Carson, and T. Delacy, "Developing a Business Information Data Warehouse for the Australian Tourism Industry: A Strategic Response," in Proc. of the International Conference on Information and Communication Technologies in Tourism 2000, New York: Springer-Verlag, 2000, pp. 147-156.

[12] J. Zabel, D. Bönke, and P. Constanta, "Open Network for Tourism (OnTour): A Concept for Electronic Commerce in the Business Processes of the Tourism Industry," in Proc. of the International Conference on Information and Communication Technologies in Tourism 2000, New York: Springer-Verlag, 2000, pp. 283-292.

[13] E. Crichton and A. J. Frew, "Usability of Information and Reservations Systems: Theory vs. Practice?" in Proc. of the International Conference on Information and Communication Technologies in Tourism 2000. New York: Springer-Verlag, 2000, pp. 408-417.

[14] R. Daniele, N. Mistilis, and L. Ward, "Partnership Australia's National Tourism Data Warehouse: Preliminary Assessment of a 
Destination Marketing System," in Proc. of the International Conference on Information and Communication Technologies in Tourism 2000, New York: Springer-Verlag, 2000, pp. 353-364.

[15] M. Friel, A. Sombert, and B. Crimes, "Service Dimensions of Travel Distribution: An Indian Case Study," in Proc. of the International Conference on Information and Communication Technologies in Tourism 2000. New York: Springer-Verlag, 2000, pp. 200-208.

[16] B. Pan and D. R. Fesenmaier, "Exploring Caller Dialogue: Analyzing Directed Information Search for Tourism Products," In Proc. of the International Conference on Information and Communication Technologies in Tourism 2001, New York: Springer-Verlag, 2001, pp. 244-250.

[17] B. Pan and D. R. Fesenmaier, "Semantics of Tourism and Travel Information Search on the Internet," in Proc. of the International Conference on Information and Communication Technologies in Tourism 2002, New York: Springer-Verlag, 2002, pp. 320-328.

[18] B. Pan and D. R. Fesenmaier, "Travel Information Search on the Internet: An Exploratory Study," in Proc. of the International Conference on Information and Communication Technologies in Tourism 2003, New York: Springer-Verlag, 2003, pp. 242-251.

[19] V. Evers, A. Kukulska-Hulme, and A. Jones, "Cross-cultural Understanding of Interface Design: A Cross-Cultural Analysis of Icon Recognition," In International Workshop on Internationalization of Products and Systems: Vol. 1, Designing for Global Markets, E. del Galdo and G. Prahbu, Eds. Rochester, NY, 1999, pp. 173-182.

[20] Y. Cho and D. R. Fesenmaier, "A Conceptual Framework for Evaluating the Effects of a Virtual Tour," in Proc. of the International Conference on Information and Communication Technologies in Tourism 2000. New York: Springer-Verlag, 2000, pp. 314-323.

[21] Bainbridge, A., "Hotel Date Entry Design and Usability," Travel UCD report, 2002. Retrieved January 2, 2005 from

http://www.travelucd.com/research/pdf/date_entry _hotel_july2002.pdf.
[22] H. Degen and S. Pedell, "JIET Design Process for E-Business Applications," In Handbook of task analysis for human-computer interaction, D.

Diaper and N Stanton, Eds. Mahwah, NJ: Lawrence Erlbaum Associates, 2003, pp. 193-220.

[23] B. Berelson. Content Analysis in Communication Research. Glencoe, IL: Free Press, 1952.

[24] N. Singh and D. W. Baack, "Web Site Adaptation: A Cross-Cultural Comparison of U.S. and Mexican Web Sites," Journal of ComputerMediated Communication, vol. 9, no. 4, 2004.

[25] D. K Farkas and J. B. Farkas, Principles of Web Design. New York, NY: Longman, 2002.
About the Authors
Quan Zhou is a Ph.D. student in the Department of Technical Communication at the University of Washington. He is interested in cross-cultural communication, digital divide, information and society, as well as telecommunication policy. $\mathrm{He}$ has published and presented in the areas of technical communication, HCI, information science as well as digital games.
Rich DeSantis is a master's student in the Department of Technical Communication at the University of Washington. He is assistant director of the Laboratory for Usability Testing and Evaluation, and prior to returning to academia, he worked in the usability field with Alucid Solutions in Atlanta, GA. His research interests are customer experience, usability, eye tracking and qualitative data analysis. He currently works in the hardware user experience group at Microsoft. 